\title{
Crossover from tunneling to incoherent (bulk) transport in a correlated nanostructure
}

\author{
J. K. Freericks \\ Department of Physics, Georgetown University, Washington, D.C. 20057-0995, U.S.A.
}

\begin{abstract}
We calculate the junction resistance for a metal-barrier-metal device with the barrier tuned to lie just on the insulating side of the metal-insulator transition. We find that the crossover from tunneling behavior in thin barriers at low temperature to incoherent transport in thick barriers at higher temperature is governed by a generalized Thouless energy. The crossover temperature can be estimated from the low temperature resistance of the device and the bulk density of states of the barrier.

PACS numbers: 73.63.-b, 71.30.+h, 71.27.+a
\end{abstract}

Many electronic devices employ quantum-mechanical tunneling in determining their transport properties. Examples include Josephson junctions $\frac{1}{\underline{1}}$ and magnetic tunnel junctions ${ }^{2}$. When designing a device manufacturing process, or when optimizing the operational characteristics of a device, it is important to have diagnostic tools that can determine if the transport is via tunneling or via defects in the barrier (such as pinholes). In superconductor-based devices, this is well understood, and was described in detail by Rowel ${ }^{3}{ }^{3}$ in the 1970's. However, the criteria relied on testing the device in the superconducting state. Interest in this problem for normal metals and for higher device operating temperatures has been driven by recent activity in magnetic tunnel junctions ${ }^{2}$. A number of useful criteria for tunneling ${ }^{4}$ have emerged for these normal-metal-based devices: (i) the junction resistance should increase with decreasing temperature; (ii) the fit of an $I V$ characteristic to a Simmons mode $1^{5}$ should have a barrier height that does not decrease and a fitted thickness that does not increase as $T$ decreases; and (iii) the junction noise should not increase at finite bias. It has also been well established that the naive criterion for tunneling, that the resistance increases exponentially with the barrier thickness is insufficient, since a rough interface plus pinholes will also yield this exponential dependence ${ }^{6}$.

In this contribution, we perform a theoretical analysis of tunneling through a correlated barrier to investigate the crossover from a tunneling regime, where transport is dominated by quantum processes that provide "shorts" across the barrier, to an incoherent bulk transport regime, where the transport occurs via incoherent thermal excitations of carriers in the barrier. In the latter case, one expects the junction resistance to scale linearly with the barrier thickness, with the slope proportional to the bulk resistivity of the barrier (which has a strong temperature dependence in an insulator). As the barrier is made thinner (or the temperature is decreased), the direct quantum-mechanical coupling of the metallic leads through states localized in the barrier begins to dominate the transport process, and the resistance is reduced from that predicted by the incoherent transport mechanism to a relatively temperature independent tunnelingbased resistance. Since the wavefunctions that connect the two metallic leads decay exponentially in the bar- rier, the tunneling resistance depends exponentially on the barrier thickness. Most commercial devices operate in this tunneling regime because the junction resistance is low enough to generate reasonable current values for low voltages and because the weak temperature dependence simplifies variations of the device parameters with temperature.

In conventional tunneling devices, which use an insulator with a large energy gap (like $\mathrm{AlO}_{x}$ ), one cannot see the crossover to the bulk transport regime, because it occurs at too high a temperature, or for too resistive junctions to be of interest. But there has been recent work in examining barriers that are tuned to lie closer to the metal-insulator transition ${ }^{7}$ (like $\operatorname{Ta}_{x} \mathrm{~N}$ ), and thereby have much smaller "energy gaps". Barriers of this type may be easier to work with because they can be made thicker and thereby be less susceptible to pinhole formation. They also can be advantageous for different applications. As the energy gap of the barrier material is made smaller (or equivalently, if the barrier potential height is reduced), it becomes possible to observe and study the crossover from tunneling to bulk transport.

We consider a device constructed out of stacks of infinite two-dimensional planes stacked in registry on top of each other. This kind of inhomogeneous layered device can be used to describe a wide range of different multilayer-based structures. We couple a bulk ballistic semi-infinite metal lead to thirty self-consistent ballistic metal planes; then we stack 1 to 20 barrier planes and then top with another thirty self-consistent ballistic metal planes followed by another bulk ballistic semiinfinite metal lead. The ballistic metal is described by a simple hopping Hamiltonian with no interactions. The barrier is described by a spin-one-half Falicov-Kimball model ${ }^{8}$ with the same hopping parameters as the metal plus strong scattering that yields correlations for the electron motion. The Hamiltonian is

$$
\mathcal{H}=-t \sum_{\langle i, j\rangle \sigma} c_{i \sigma}^{\dagger} c_{j \sigma}+\sum_{i \sigma} U_{i}^{F K} w_{i}\left(n_{i \sigma}-\frac{1}{2}\right),
$$

where $c_{i \sigma}^{\dagger}\left(c_{i \sigma}\right)$ creates (destroys) a conduction electron at site $i$ with spin $\sigma$ and $t$ is the hopping parameter. The hopping is on a simple cubic lattice constructed from the stacked two-dimensional planes; i.e., the hopping integral 
is chosen to be the same within a plane and between two planes. $U_{i}^{F K}$ is the Falicov-Kimball interaction and $w_{i}$ is a classical variable, equal to zero or one, which denotes the presence of a scatterer at site $i$. Finally, $n_{i \sigma}=c_{i \sigma}^{\dagger} c_{i \sigma}$ is the electron number operator. The Falicov-Kimball interaction is nonzero only within the barrier, where we set it equal to $6 t$-large enough to create an insulator with a gap of $0.4 t$. The average concentration of scatterers is $\left\langle w_{i}\right\rangle=1 / 2$ and we choose half filling for the electrons as well (with our choice of interaction, this corresponds to a vanishing chemical potential). In order to be quantitative, we pick the hopping parameter to satisfy $t=0.25 \mathrm{eV}$, which yields a bandwidth of $3 \mathrm{eV}$ for the metallic leads and a gap of $100 \mathrm{meV}$ for the correlated insulator (much smaller than a conventional oxide insulator). We solve for the Green's functions using an inhomogeneous dynamical mean field theory calculation described elsewhere $e^{9,10,11,12}$. The resistance-area product for this device is calculated by a real-space version of Kubo's formula. We take the lattice constant to be $0.3 \mathrm{~nm}$.

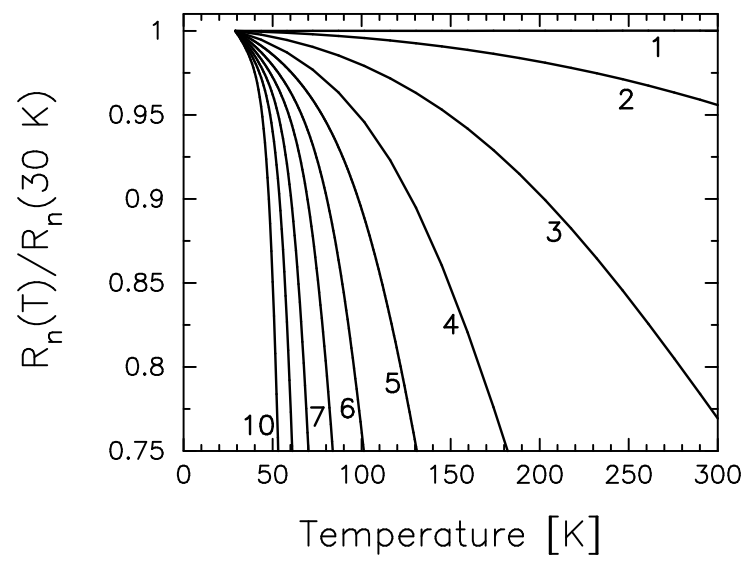

FIG. 1: Ratio of the resistance of the junction at temperature $T$ to the resistance at $30 \mathrm{~K}$. The different curves correspond to different thicknesses of the barrier, which are labeled with an integer denoting the number of atomic planes in the barrier. As expected, the temperature dependence of the resistance increases as the barrier is made thicker, because the barrier is becoming more bulk-like. However, in this regime, all of the transport is still dominated by tunneling.

In Fig. 1 we plot the ratio of the junction resistance at temperature $T$ to the resistance at $30 \mathrm{~K}$ for junctions with a barrier thickness ranging from 1 to 10 atomic planes. In all cases, the resistance shows a weak temperature dependence with an insulator-like character. This low- $T$ behavior is often used as a diagnostic to indicate that tunneling is occuring in a junction ${ }^{4.13}$, and that certainly is the case here. Note how the temperature dependence increases as the thickness increases. This is because the thicker the barrier is, the more it looks like a bulk material, and an insulating barrier has strong (exponentially activated) temperature dependence in the bulk.

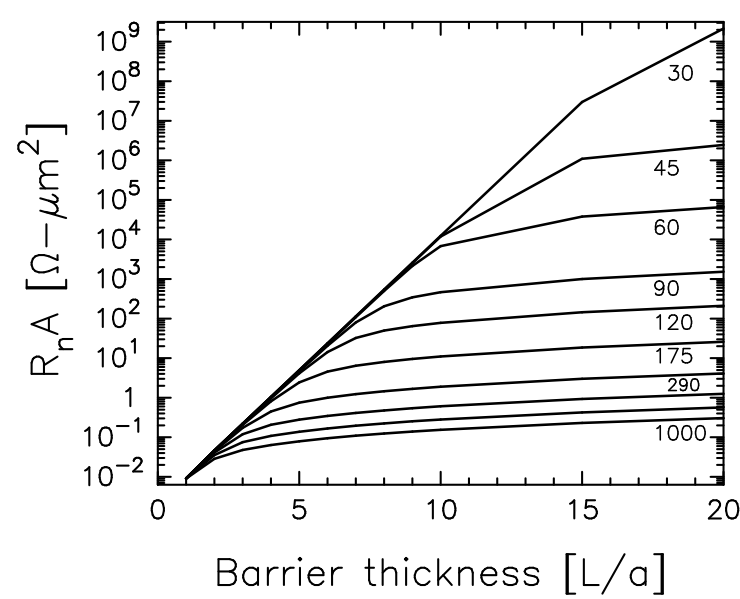

FIG. 2: Resistance-area product as a function of the barrier thickness $L$ for a number of different temperatures (the labels on the curves are in K). Notice how the thin barriers have an exponential dependence on thickness, which gives way to a linear dependence as the junctions are made thick enough. This crossover region moves to thinner barriers as the temperature is increased.

Since our junctions are defect free, with atomically smooth interfaces, we can analyze the resistance at fixed temperature as a function of the barrier thickness to look for exponential dependence in the tunneling regime, with a crossover to linear dependence in the incoherent (bulk) transport regime. This is plotted in Fig. 2 for a number of different temperatures, ranging from $30 \mathrm{~K}$ to $1000 \mathrm{~K}$. Note how we see a perfect exponential dependence on thickness for thin barriers, which then gives way to a crossover to linear behavior as the junctions are made thicker and the transport becomes incoherent and thermally activated. Because of the thermal activation, this crossover moves to thinner barriers as the temperature is increased. But it is interesting to note that there is no simple relationship between the bulk gap (approximately $50 \mathrm{meV}$ or $550 \mathrm{~K}$ when measured from the $T=0$ chemical potential) and the location of the crossover thickness as a function of temperature. Indeed, as $T$ is increased, this crossover region is pushed to thinner and thinner barriers. This type of behavior has been seen in Josephson junctions made from high temperature superconductors using molecular-beam-epitaxy ${ }^{14}$. When the barrier was increased from 1 to 3 to 5 to 7 atomic planes, the junction resistance initially increased exponentially, and then started to turn over to a more linear dependence on thickness. However, because the high-temperature superconductor is a d-wave superconductor, there is strong temperature dependence to the junction resistance, even in the tunneling regime, so direct comparison with results given here is impossible. This behavior has also been seen in some magnetic tunnel junctions ${ }^{15}$ where an exponential increase as a function of thickness gives way to an essentially constant dependence on thickness for thicker Aluminum regions. What is less known about this data 
is how much of the Aluminum is oxidized in the manufacturing process. Also, no temperature scans at fixed thickness were reported.

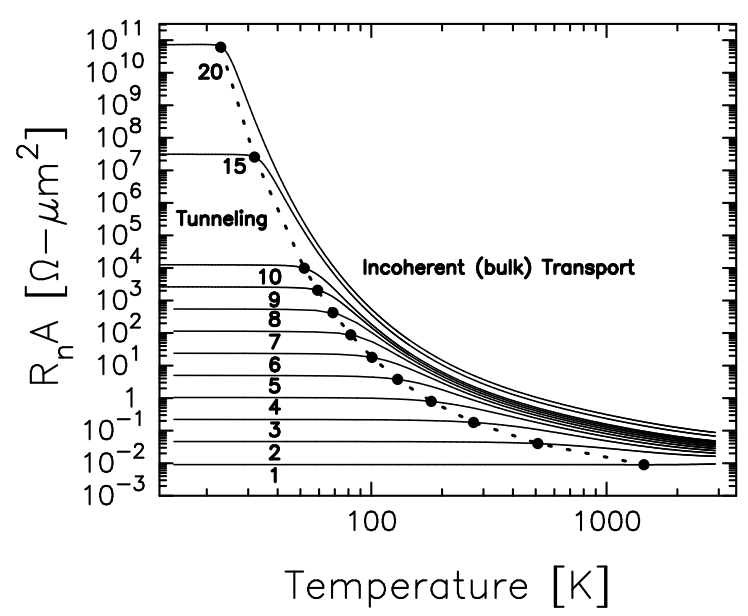

FIG. 3: Resistance-area product as a function of temperature for a number of different barrier thicknesses plotted on a log-log plot. Notice how the thin barriers have a weak dependence on temperature, and a constant step-size increase in the logarithm of the resistance as the thickness increases, indicating tunneling behavior, and how there is a crossover to incoherent transport as $T$ is increased. The dashed line shows the boundary where the generalized Thouless energy is equal to $k_{B} T$. This marks the approximate crossover from tunneling (for $E_{\mathrm{Th}}(T) \gg k_{B} T$ ) and incoherent transport (for $\left.E_{\mathrm{Th}}(T) \ll k_{B} T\right)$.

In Fig. 3, we plot $R_{n}(T) A$ versus $T$ for a variety of barrier thicknesses on a log-log plot. This figure clearly shows the tunneling regime, where the resistance-area product is approximately constant, and it shows the incoherent regime, where the resistance-area product has a strong temperature dependence. The dashed line, that divides these two regions is an approximate boundary that denotes the crossover region for the two different types of transport. This crossover line is determined by equating an energy scale extracted from the resistance with the temperature. When this energy scale is larger than $k_{B} T$ we have tunneling, when it is lower than $k_{B} T$ we have incoherent transport. The energy scale is a generalized Thouless energy ${ }^{16}$, valid for a barrier that is described by an insulator that does not have either ballistic or diffusive transport. The generalized Thouless energy $E_{T h}$ is the energy scale constructed from the resistance at temperature $T$ via the expression

$$
E_{\mathrm{Th}}(T)=\frac{\hbar}{R_{n}(T) \frac{2 e^{2}}{\hbar} \int d \omega\left(-\frac{d f(\omega)}{d \omega}\right) \rho_{\text {int }}(\omega) L}
$$

where $f(\omega)=1 /\left[1+\exp \left(\omega / k_{B} T\right)\right]$ is the Fermi-Dirac distribution, $\rho_{\text {int }}(\omega)$ is the bulk density of states in the insulator, and $L$ is the barrier thickness. This definition of $E_{\mathrm{Th}}$ agrees with the conventional notion of $\hbar / t_{d w e l l}$, relating the Thouless energy to the dwell time in the barrier, when the transport in the barrier is described by either a ballistic metal (where the Thouless energy varies like $C / L$ ) or a diffusive metal (where the Thouless energy varies like $C / L^{2}$ ), but it can now be generalized for an insulating barrier as well (where the Thouless energy now picks up a substantial temperature dependence).

The notion of a Thouless energy can be employed as a diagnostic for tunneling devices. Since $R_{n}(T)$ depends weakly on $T$ in the tunneling regime, one can measure $R_{n}$ at low $T$, and estimate the crossover temperature, by computing a simple integral over the bulk insulator density of states. Then one evaluates $E_{\mathrm{Th}}(T)$ employing Eq. (2) using the low-temperature value of the resistance. The crossover temperature is estimated by the point where $E_{\mathrm{Th}}(T)=k_{B} T$. Note further that this crossover temperature is not proportional to the gap of the bulk insulator, but rather is a complicated function of the barrier thickness, and the strength of the correlations.

In summary, we have determined an energy scale extracted from the resistance of a junction, that governs the crossover from tunneling to incoherent transport. This energy scale approaches zero as the barrier thickness becomes large, hence it could have applicability to any tunneling-based device, but when we examine the common resistance-area products of actual devices, it becomes clear that this concept will have the most applicability to junctions with barriers tuned to lie close to the metal-insulator transition. Since it is possible such devices will be used for devices of the future, the concept of a generalized Thouless energy should become an important diagnostic tool in evaluating the quality of devices, and allow one to engineer the thickness and operating temperature range to guarantee tunneling with the chosen barrier.

Acknowledgments: We acknowledge support from the National Science Foundation under grant number DMR0210717 and from the Office of Naval Research under grant number N00014-99-1-0328. High performance computer time was provided by the Arctic Region Supercomputer Center and the U. S. Army Engineering Research and Development Center. We also acknowledge useful discussions with J. Eckstein, B. Jones, N. Newman, B. Nikolić, S. Parkin, J. Rowell, I. Schuller and S. Shafraniuk.
1 B. D. Josephson, Phys. Lett. 1, 251 (1962).

2 J. S. Moodera, L. R. Kinder, T. M. Wong, and R. Meser- vey, Phys. Rev. Lett. 74, 3273 (1995); T. Miyazaki and N. Tezuka, J. Magn. Magn. Mater. 139, L231 (1995); S. 
Tehrani, J. M. Slaughter, E. Chen, M. Durlam, J. Shi, and M. DeHerrera, IEEE Trans. Magn. 35, 2814 (1999); J. Zhang, Data Storage 5, 31 (1998); S. A. Wolf and D. Treger, IEEE Trans. Magn. 36, 2748 (2000).

3 J. M. Rowell, in Tunneling Phenomena in Solids, edited by E. Burnstein and S. Lundqvist (Plenum, New York, 1969), p. 273.

4 J. J. Akerman, J. M. Slaughter, R. W. Dave, and I. K. Schuller, Appl. Phys. Lett. 79, 3104 (2001).

5 J. G. Simmons, J. Appl. Phys. 34, 1793 (1963).

6 D. A. Rabson, B. J. Jönsson-Akerman, A. H. Romero, R. Escudero, C. Leighton, S. Kim, and I. K. Schuller, J. Appl. Phys. 89, 2786 (2001); W. H. Rippard, A. C. Perrella, F. J. Albert, and R. A. Buhrman, Phys. Rev. Lett. 88, 046805 (2002); L. S. Dorneles, D. M. Schaefer, M. Carara, and L. F. Schelp, Appl. Phys. Lett. 82, 2832 (2003).

7 A.B. Kaul, S.R. Whitely, T. van Duzer, L. Yu, N. Newman, and J.M. Rowell, Appl. Phys. Lett. 78, 99 (2001).

8 L. M. Falicov and J. C. Kimball, Phys. Rev. Lett. 22, 997 (1969).

9 M. Potthoff and W. Nolting, Phys. Rev. B 59, 2549 (1999).
10 P. Miller and J. K. Freericks, J. Phys.: Conden. Matter 13, 3187 (2001).

11 J.K. Freericks, B.K. Nikolić, and P. Miller, Phys. Rev. B 64, 054511 (2001); Phys. Rev. B 68, 099901 (E) (2003).

12 J.K. Freericks, B.K. Nikolić, and P. Miller, Int. J. Mod. Phys. B 16, 531-561 (2002).

13 U. Rüdiger, R. Calarco, U. May, K. Samm, J. Hauch, H. Kittur, M. Sperlich, and G. Güntherodt, J. Appl. Phys. 89, 7573 (2001).

14 J. N. Eckstein and I. Bozovic, Ann. Rev. Mater. Sci. 25, 679 (1995).

15 M. Covington, J. Nowak, and D. Song, Appl. Phys. Lett. 76, 3965 (2000).

16 J. T. Edwards and D. J. Thouless, J. Phys. C 5, 807 (1972); D. J. Thouless, Phys. Rep. 13, 93 (1974); A. Atland, Y. Gefen, and G. Montambaux, Phys. Rev. Lett. 76, 1130 (1996); M. Janssen, Fluctuations and Localization in Mesoscopic Electron Systems, (World Scientific, Singapore, 2001). 